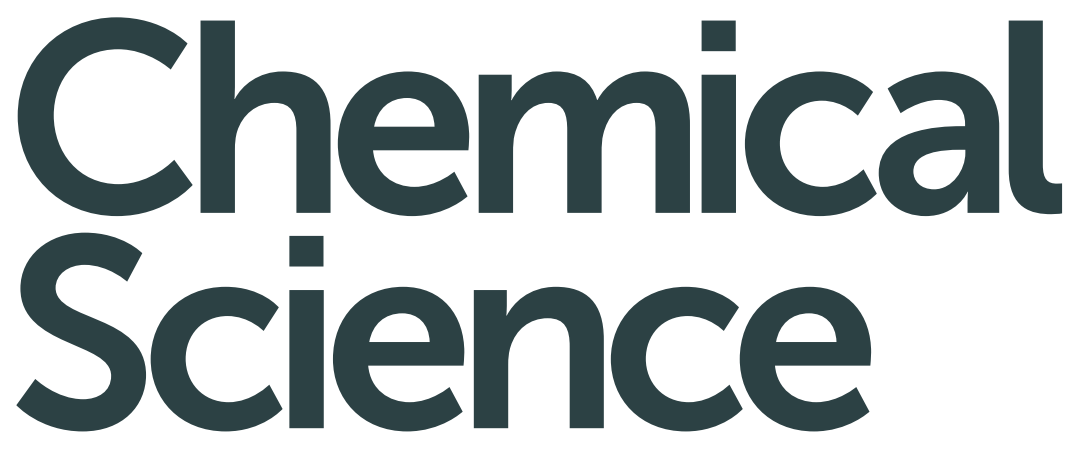

rsc.li/chemical-science
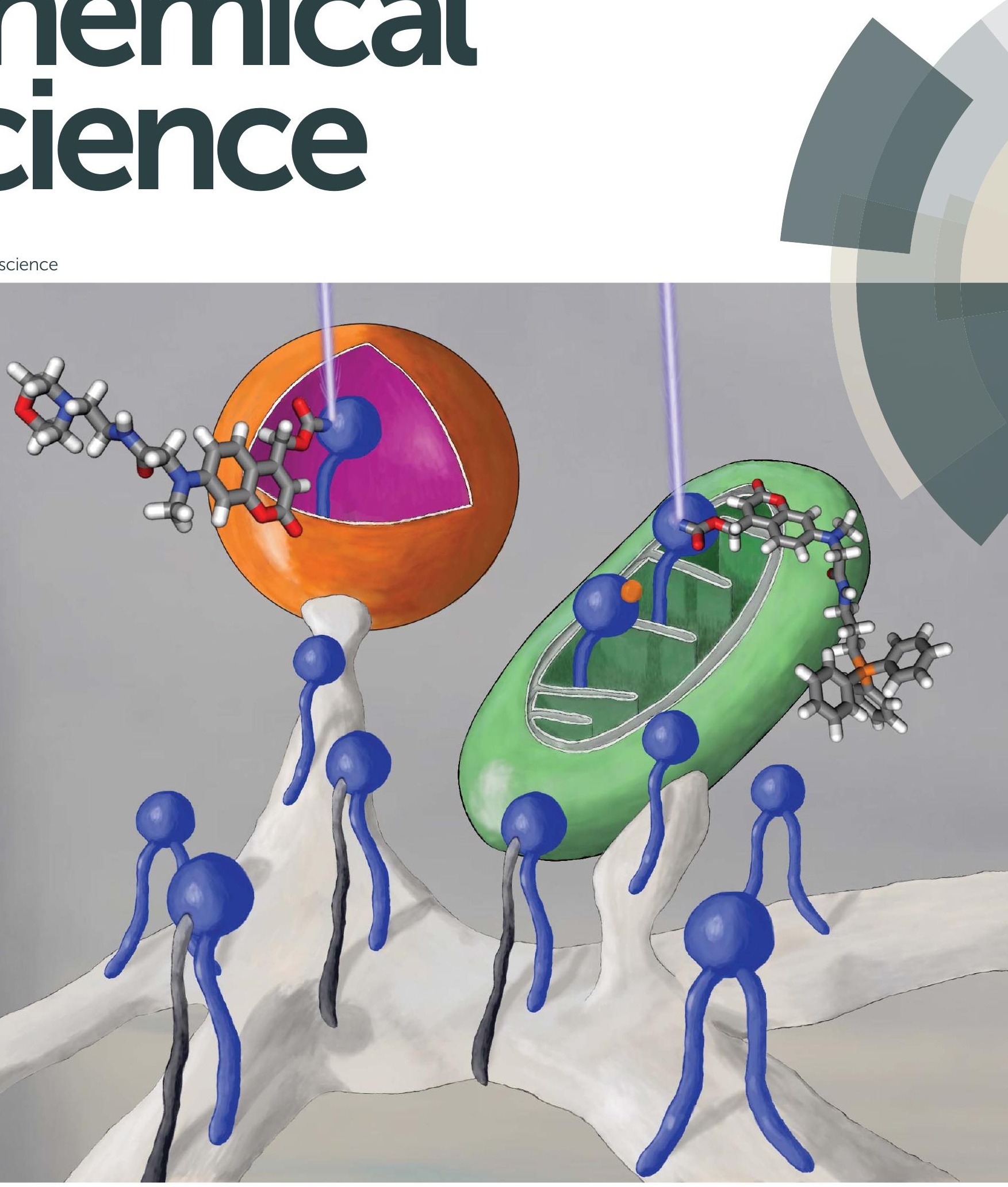

ISSN 2041-6539

ROYAL SOCIETY | Celebrating OF CHEMISTRY | IYPT 2019
EDGE ARTICLE

Howard Riezman et al.

Lysosome-targeted photoactivation reveals local

sphingosine metabolism signatures 
Check for updates

Cite this: Chem. Sci., 2019, 10, 2253

๑ All publication charges for this article have been paid for by the Royal Society of Chemistry

Received 14th August 2018

Accepted 21st December 2018

DOI: $10.1039 / \mathrm{c} 8 \mathrm{sc} 03614 \mathrm{~d}$

rsc.li/chemical-science

\section{Lysosome-targeted photoactivation reveals local sphingosine metabolism signatures $\dagger$}

\author{
Suihan Feng, (iD a Takeshi Harayama, (iD a Dalu Chang, ${ }^{\mathrm{b}} \mathrm{J}$. Thomas Hannich, (D) a \\ Nicolas Winssinger (iD ${ }^{\mathrm{b}}$ and Howard Riezman (iD *a
}

\begin{abstract}
Lipids are essential components of eukaryotic cell membranes and play crucial roles in cellular signaling and metabolism. While increasing evidence shows that the activities of lipids are dependent upon subcellular localization, tools to study local lipid metabolism and signaling are limited. Herein, we report an approach that enabled us to selectively deliver photo-caged lipids into lysosomes and thereafter to quickly release the lipid molecules by illumination. On combining this method with genetic techniques and lipidomics, we were able to investigate the localization-dependent metabolism of an important intermediate of sphingolipid metabolism, sphingosine. Our data reveal a distinct metabolic pattern of lysosomal sphingosine. In general, this method has the potential to serve as a platform to study lysosomal metabolism and signaling of various lipids and metabolites in living cells.
\end{abstract}

\section{Introduction}

Many lipid molecules are metabolic intermediates in lipid metabolic pathways, but they are also second messengers and essential components of cell signaling networks, involved in a variety of physiological and pathological processes. A comprehensive understanding of their activities often requires quantitative analysis of a large number of relevant lipid molecules in a metabolic pathway by mass spectrometry. To this end, we have previously established an unbiased systematic lipidomics approach in combination with gene deletions, which was used to identify novel regulatory mechanisms in yeast cells and show that lipid profiles are indicative of genetic makeup. ${ }^{1}$ While removing key metabolic enzymes can dramatically alter specific lipid levels and thus serve as a powerful approach, it lacks temporal resolution and is not suitable for controlling signaling lipids which are transiently produced and have very short half-lives. In addition, these mutants generally affect whole classes of lipids rather than individual species.

To quickly generate signaling lipids in living cells, photoactivation ("uncaging") has been introduced into the lipid field, which can release a large number of lipid molecules to trigger specific lipid-mediated signaling pathways. ${ }^{2}$ Typically, a photocleavable protecting group is pre-installed on the head group of a lipid molecule to block its activity. Once in the cells, UV illumination quickly removes the photo-protecting group and

${ }^{a}$ Department of Biochemistry, NCCR Chemical Biology, University of Geneva, Quai Ernest-Ansermet 30, 1211, Geneva, Switzerland. E-mail: howard.riezman@unige.ch ${ }^{b}$ Department of Organic Chemistry, NCCR Chemical Biology, University of Geneva, Quai Ernest-Ansermet 30, 1211, Geneva, Switzerland

$\dagger$ Electronic supplementary information (ESI) available. See DOI: $10.1039 / \mathrm{c} 8 \mathrm{sc} 03614 \mathrm{~d}$ thereby releases the lipid messenger in a non-invasive manner. Due to the usefulness of the high temporal resolution obtained by uncaging, a number of lipids have been successfully caged using this technology. ${ }^{3-6}$ In a complementary strategy, photoswitchable lipid analogues have been used to artificially manipulate lipid activities but may require mechanistic knowledge of protein-lipid interactions, and the metabolism may be compromised due to the structural differences with the native lipid. ${ }^{7,8}$ Surprisingly, most of these chemical tools were developed to explore the signaling functions of lipids rather than studying lipid metabolism.

In addition to the temporal resolution that is essential to study lipid signaling and metabolism, lipid functions are also highly associated with subcellular localization. However, most caged lipids lack subcellular specificity. In order to dissect the compartmentalized lipid metabolism, we have developed a mitochondria-specific photoactivation method, which allowed us to monitor mitochondrial sphingosine metabolism and signaling in living cells directly (Fig. 1B). ${ }^{9}$ We have provided experimental evidence that lipid metabolism is highly dependent on the subcellular localization. Further understanding of local lipid metabolism requires novel tools that can release lipids in various organelles and other locations in cells.

Among the membrane-bound organelles, lysosomes are acidic compartments with the primary role of mediating the catabolism of proteins, polysaccharides, and complex lipids. They are highly dynamic and are involved in membrane trafficking including secretion, endocytosis, phagocytosis, and autophagy. Since lysosomes are the central hubs of macromolecule degradation, their activities are tightly regulated by the nutrient status and cellular signaling. ${ }^{10}$ Conversely, lysosomal dysfunction is responsible for a wide range of diseases that 
A

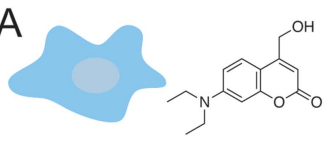

Global uncaging
B

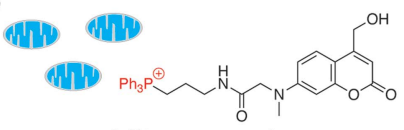

Mito- uncaging

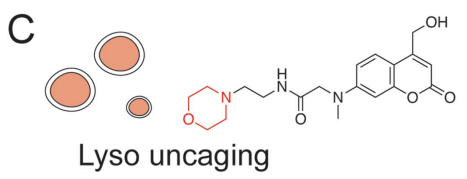

Fig. 1 Chemical structures of coumarin-based photocleavable cage molecules: (A) diethylaminohydroxyl coumarin without obvious intracellular selectivity; (B) triphenylphosphonium (TPP) cationcoupled coumarin specifically targets to mitochondrial matrix; (C) morpholino coumarin specifically targets to lysosomes.

show abnormal accumulation of metabolites. For example, Niemann-Pick type C1 (NPC1) disease is a neurodegenerative lysosomal storage disorder, where sphingosine, cholesterol, and other lipids have been found to accumulate inside the acidic stores of cells. ${ }^{11}$

To systematically investigate the local lipid metabolism, we introduce here a lysosome-targeted photoactivation method by coupling the coumarin molecule to a morpholino group (Fig. 1C). In this way, we were able to accumulate caged sphingosine and cholesterol inside lysosomes in living cells. More importantly, use of an isotope labeled $\mathrm{D}_{7}$-sphingosine allowed us to track its conversion to ceramides in living cells. By combining this method with CRISPR/Cas9 genome modification we can directly compare the metabolic fate of sphingosine that was released from distinct subcellular locations.

\section{Results and discussion}

Replacing the triphenylphosphonium cation with a morpholino substituent (Scheme 1) on the alkyl chain protruding from the 7-amino group of coumarin led to our target compound 1. Coupling to sphingosine using previously described protocols afforded Lyso-So. ${ }^{9}$ Lyso-Chol was conveniently synthesized by coupling 1 with cholesteryl chloroformate. Our data show that, upon UV illumination, Lyso-So was quickly uncaged to give free sphingosine within two minutes. Similar results were also observed for Lyso-Chol (Fig. S1†).
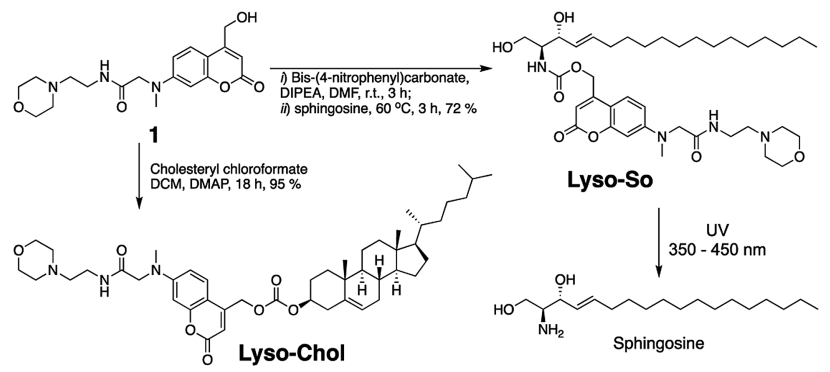

Scheme 1 Synthesis of lyso-caged sphingosine (Lyso-So) and cholesterol (Lyso-Chol).
Next, we sought to determine the subcellular localization of the lyso-caged compounds in living cells. Using HeLa cells, we incubated the Lyso-So $(5 \mu \mathrm{M})$ together with LysoTracker for 15 min and washed. The fluorescence staining of Lyso-So overlaps well with the LysoTracker, indicating that the Lyso-So is mainly targeted to the lysosomes in living cells (Fig. 2). Lysosomes maintain acidic environments between $\mathrm{pH} 4$ and $\mathrm{pH}$ 5. To test whether the fluorescence of the coumarin-based probes is $\mathrm{pH}$ sensitive, we measured the fluorescence intensity of Lyso-So in different $\mathrm{pH}$ buffers and found that the fluorescence was only slightly increased at pH 4.0 (Fig. S2†). Therefore, this small difference cannot account for the increased fluorescence intensity from the lysosomes in the living cells and we confirm that most of the Lyso-So is co-localized well with LysoTracker (Fig. 2B and C). In preliminary experiments we noticed that washing steps are necessary in order to remove fluorescence staining outside lysosomes, probably caused by the hydrophobic lipid chain of the caged molecule. Importantly, similar fluorescence staining was also found with the caged cholesterol compound (Fig. S3†), suggesting that the caging group has the potential to target a broad range of caged lipids to lysosomes.

Since the lysosomes are enriched with a variety of hydrolases and lipases required for their metabolic activity and specific conditions prevail in their interior, it is crucial to determine whether the caged lipids are stable prior to UV illumination. For this purpose, we prepared a caged lipid using an isotope labelled $\mathrm{D}_{7}$-sphingosine as a precursor, which ensures that the photo-released sphingosine is easily distinguished from the endogenous sphingolipids by mass spectrometry. Next, we applied the $\mathrm{D}_{7}$-labelled caged sphingosine in HeLa cells under different experimental conditions. After collecting the cells and extracting the lipids, the samples were analysed by LC-MS. We detected a significant increase of $\mathrm{D}_{7}$-sphingosine after UV illumination of the $\mathrm{D}_{7}$-Lyso-So treated cells, and a small amount of $\mathrm{D}_{7}$-sphingosine in the cells treated with Lyso-So in the absence

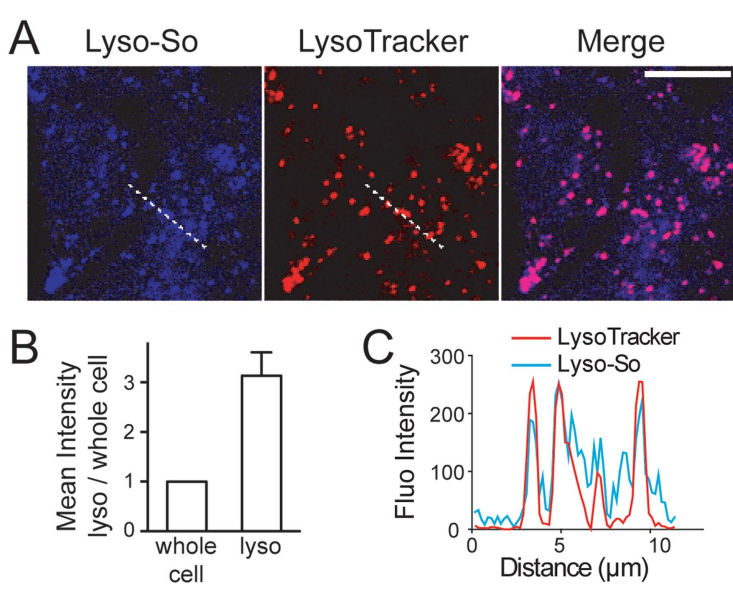

Fig. 2 Fluorescence staining of Lyso-So: (A) representative fluorescence images of HeLa cells stained with Lyso-So $(5 \mu \mathrm{M})$ and LysoTracker Red DND-99 (100 nM). Scale bar: $10 \mu \mathrm{m}$. (B) Mean intensities of lysosomes versus the whole cells. Error bars represent SEM, $n=10$ cells (see detailed description in the ESI $\dot{T}$ ). (C) Intensity profiles of the white dotted lines in Lyso-So and LysoTracker channels, respectively. 
A

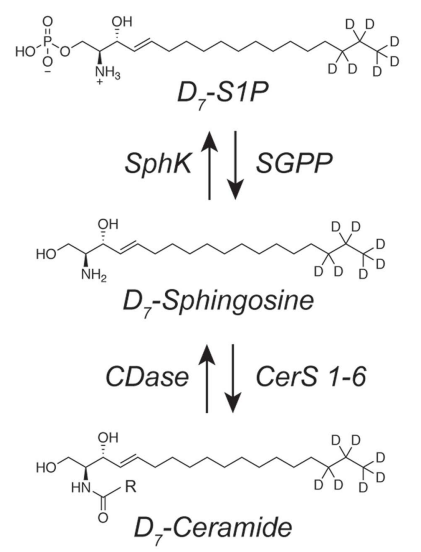

B
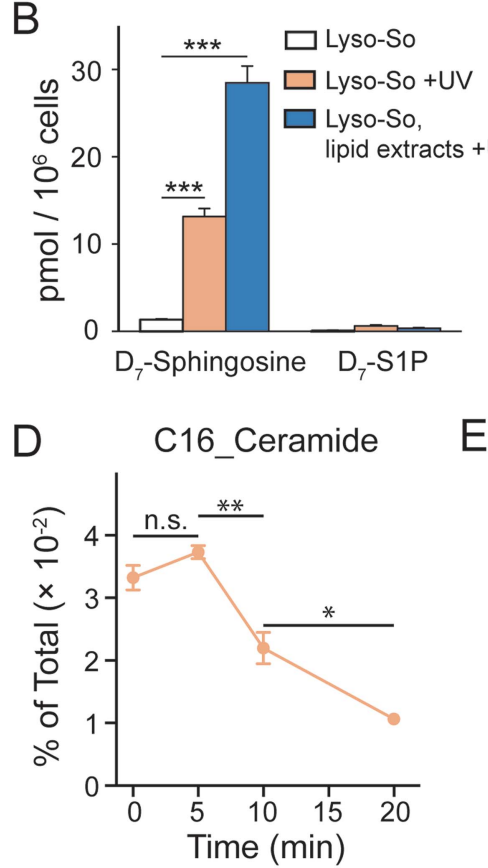

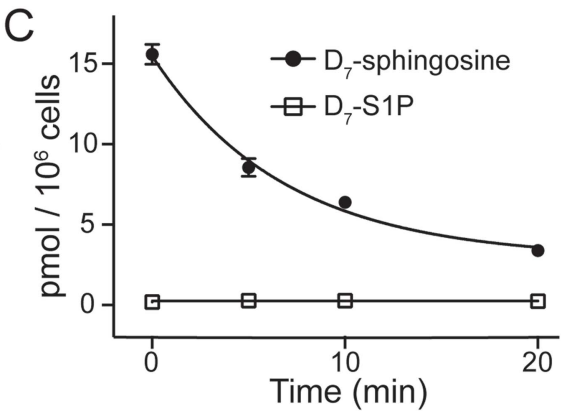

$E$

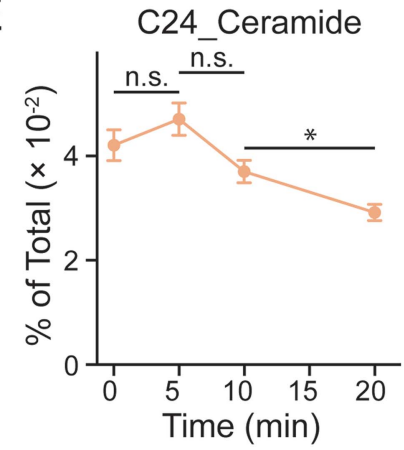

Fig. 3 Sphingolipid analysis of lysosome-targeted caged sphingosine. (A) Schematic illustration of the interconversion between sphingosine, sphingosine 1-phosphate (S1P) and ceramides. Sphingosine can be phosphorylated into S1P by sphingosine kinases (SphKs); and S1P can be dephosphorylated into sphingosine by sphingosine 1-phosphate phosphatases (SGPPs). Ceramide synthases (CerS 1-6) convert sphingosine into ceramides with various acyl chain lengths; ceramidases (CDases) cleave ceramides to yield sphingosine and fatty acids. (B) Analysis of $D_{7}{ }^{-}$ sphingosine and $D_{7}$-S1P levels under various experimental conditions as indicated. (C) Changes of sphingosine and S1P levels over time after uncaging of $D_{7}$-Lyso-So in HeLa cells. The decay curves were generated by fitting into a single phase decay function. Data in (B) and (C) were normalized with respect to the amount of $C 17$ internal standards and cell numbers. ( $D$ and $E$ ) Metabolism of newly formed $D_{7}$-ceramides after photo-releasing $D_{7}$-sphingosine from Lyso-So in HeLa cells. Data in (D) and (E) were normalized by the sum of all measured lipid signals and presented as "mol\% of total". All data represent the average of three independent experiments. Error bars represent SEM. *p<0.05, ** $p<0.01$, $* * * p<0.001$, n.s., not significant, Students' $t$-test.

of UV light (Fig. 3B). Interestingly, when the cells were treated with $\mathrm{D}_{7}$-Lyso-So, processed for extraction, and afterwards exposed to UV illumination, we detected twice the amount of sphingosine compared to that when uncaging in the living cell (Fig. 3B, Lyso-So, lipid extracts + UV vs. Lyso-So + UV). The different levels of sphingosine could result from the rapid metabolic turnover of the released sphingosine in the cells during the uncaging process or could be due to the metabolism of the released sphingosine prior to uncaging. In mammalian cells, sphingosine can be converted into two products, sphingosine 1-phosphate (S1P) and ceramides (Fig. 3A). Surprisingly, only a minimal S1P signal was observed after lysosomal uncaging, in contrast to our previous findings that a significant amount of sphingosine was quickly phosphorylated into S1P after being released inside mitochondria. ${ }^{9}$ Moreover, in the time-course experiment, we observed the decline of $\mathrm{D}_{7}$-sphingosine levels over time, but the $\mathrm{D}_{7}$-S1P levels remained constant at the basal level (Fig. 3C), suggesting that sphingosine was not metabolized through the S1P degradation pathway from lysosomes.

The second metabolic fate is the formation of ceramides via ceramide synthases. As ceramides are much more abundant compared to sphingosine, the use of $\mathrm{D}_{7}$-caged sphingosine offers an advantage of capturing the changes of the newly formed ceramides separately from the endogenous ceramide pool by mass spectrometry. We treated cells with $\mathrm{D}_{7}$-Lyso-So in the absence and presence of UV illumination and then extracted lipids for lipidomics analysis. To our surprise, a significant amount of $\mathrm{D}_{7}$-ceramides was detected without uncaging (Fig. S4†), suggesting the partial decomposition of the Lyso-So. By comparison, in cells that were treated with mitochondriatargeted caged sphingosine (Mito-So) ${ }^{9}$ or globally caged sphingosine (Sph-Cou), ${ }^{6}$ only very low ceramide levels were detected without uncaging (Fig. S4†). Since sphingosine and S1P levels were measured using LC-MS and ceramide levels were measured via direct infusion and MS/MS analysis, it is difficult to directly compare the data and estimate how much of the caged sphingosine was released prior to UV illumination. Nevertheless, the sphingosine level in the live-cell uncaging experiment (Fig. 3B, left) suggests that a large portion of sphingosine was still intact before uncaging. As the three caged probes share similar chemical structures and only Lyso-So showed obvious premature release, the decomposition of LysoSo takes place in lysosomes and is mostly due to the enzymatic activities or the specific environment inside the lysosomes. Thus, even though sphingosine metabolism starts to occur before illumination, it still reflects the metabolic features of lysosomes. It is worth noting that a recent report also described 
caged probes that target caged lipids to lysosomes and other organelles, ${ }^{12}$ but the stability of the probes in cells prior to uncaging was not analyzed.

Although partial decomposition of the caged probe reduces our temporal control of lipid metabolism, it is clear from the results that sphingosine phosphorylation is much less important in lysosomes than in mitochondria. This could be explained in several ways. First, there is no evidence for localization of sphingosine kinase to lysosomes, in contrast to evidence that sphingosine kinase 2 (SphK2) localizes to mitochondria, ${ }^{13}$ which should facilitate localized S1P formation. Second, most of the lysosome-released sphingosine could be quickly transferred to the endoplasmic reticulum (ER) where it is acylated into ceramides via ceramide synthases. This is consistent with the notion of a sphingolipid salvage pathway in which sphingoid bases efficiently traffic to the ER to be converted into ceramides or complex sphingolipids for further distribution. ${ }^{14}$ Finally, lysosomes are known to contain nonspecific phosphatases and they could affect the amount of S1P. Degradation of S1P by the S1P lyase (SGPL) is less likely because there is not even a transient increase of S1P and the SGPL is localized in the ER. ${ }^{15}$ Taken together, our results show that the sphingosine metabolism is tightly organized in cells depending on the subcellular compartment and that lysosomal sphingosine does not freely diffuse in the cytoplasm once released from lysosomes.

We further investigated the metabolism of lysosomal sphingosine after uncaging, focusing on ceramide production. In mammalian cells, six ceramide synthases (CerS 1-6) are responsible for converting sphingosine into ceramides with different acyl chain length specificities. ${ }^{16}$ Conversely, various ceramidases (CDases) can cleave ceramides to form free sphingosine and fatty acids (Fig. 3A). ${ }^{14}$ To monitor the dynamics of ceramides, we performed a "pulse-chase" type of experiment using our uncaging assay. After photo-releasing $\mathrm{D}_{7}$-sphingosine in lysosomes, we collected the cells after different times at $37^{\circ} \mathrm{C}$ and extracted lipids for lipidomics analysis. We found that $\mathrm{C} 16$ and $\mathrm{C}_{2} 4 \mathrm{D}_{7}$-ceramides were the major sphingolipids formed and thus concentrated on the metabolism of these two ceramides in further analysis. Because a significant amount of C16 and $\mathrm{C}_{24} \mathrm{D}_{7}$-ceramides were detected without UV illumination, the $\mathrm{D}_{7}$-ceramides measured directly after uncaging ( 0 minute, Fig. 3D and E) of Lyso-So most likely reflect the initial synthesis of the metabolic products of $\mathrm{D}_{7}$-sphingosine released in lysosomes prior to the uncaging process. The amount increases after 5 minutes of chase. The results revealed a distinct metabolic profile of $\mathrm{C} 16$ and $\mathrm{C} 24$ ceramides. As shown in Fig. 3D, the C16 ceramide reached its maximum level within five minutes after uncaging and subsequently declined. In contrast, the C24 ceramide levels were similar for the first 5 minutes, but then remained more stable only decreasing after 10 minutes (Fig. 3E). Meanwhile, the endogenous ceramide levels and major lipid species remained stable over time (Fig. S5 $\dagger$ ), implying that the uncaging assay did not have an effect on the ceramide homeostasis and other major lipid species.

Previously, we have shown that depending on the subcellular compartments, ceramides are metabolized following distinct patterns. ${ }^{9}$ After releasing sphingosine in mitochondria, we recorded continuous accumulation of ceramides with C16 and C24 fatty acyl chains. Since ceramide synthases are localized in the ER, ${ }^{16}$ these unique metabolic patterns could be caused by domain differences within the ER and differential delivery dependent upon the donor compartment. Consistent with previous findings, our data here show that the lysosomereleased sphingosine was rapidly converted into ceramides which were further metabolized over time. The rapid turnover points to the possibility that the lysosome and ER are tightly connected for sphingosine transfer towards ceramide synthesis. Interestingly, the C16 and C24 ceramides exhibited different turnover rates, unlike our previous results where both C16 and C24 ceramides kept a similar metabolic pace regardless of whether the sphingosine was released from mitochondria, whole cells, or directly added from the extracellular medium. This rapid turnover might be because the C16 ceramide is a signaling molecule involved in apoptosis. ${ }^{17}$

To gain further insights into ceramide metabolism, we turned to CRISPR/Cas9 genome modification, ${ }^{18}$ a powerful way to interfere with the metabolic network. Combining the knockout cells with the site-specific uncaging methods provides a unique opportunity to explore the functions of key enzymes in the sphingolipid metabolic pathway. For this purpose, we prepared ceramide synthase 5/6 double knockout (CerS 5/6 dKO) cells that lack the major activities to convert sphingosine into the C16 ceramide. Surprisingly, we did not observe a meaningful decline of steady state C16 ceramide levels in the knockout cells (Fig. S7E †). Therefore, the cells must circumvent the deficiency of ceramide synthase $5 / 6$ via other pathways. Nevertheless, when we performed a "pulsechase" protocol releasing the caged $\mathrm{D}_{7}$-sphingosine in the cells, we detected a very strong decrease in the synthesis of the C16 $\mathrm{D}_{7}$-ceramide in the CerS 5/6 dKO cells (Fig. 4), suggesting that the conversion to the C16 ceramide via CerS 5/6 activity is strongly reduced in the knockout cells. The low amount of C16 ceramide detected in the dKO cells (Fig. 4C and D) could reflect residual enzymatic activities of CerS 5 and/or 6 (Fig. S6 $\dagger$ ) or could result from inefficient use of palmitoyl-CoA by other ceramide synthases.

Next, we investigated how subcellular localization affects the conversion of sphingosine into the different ceramide species. Using HeLa cells and the generated CerS 5/6 dKO cells, we compared the conversion to ceramide after releasing $\mathrm{D}_{7}$-sphingosine from the whole cells, mitochondria and lysosome (Fig. 4 and S7 $\dagger$ ). Since the C16 ceramide generated was quickly metabolized after 5 minutes of chase (Fig. 3C), we chose to analyse the lipid profiles at the 5 minute time point after uncaging. In wildtype cells, we detected ceramides with various acyl chains, but the major ones formed were C16 and C24 ceramides, which reached the highest level. Interestingly, lysosome-released sphingosine generated proportionally more C24 ceramide, whereas mitochondria-released sphingosine generated more C16 ceramide (Fig. 4B). When conversion to the C16 ceramide was blocked by deletion of CerS $5 / 6$, we detected a redistribution in the ceramide profile, in which C18, C24 and C26 became more prominent (Fig. 4D). Again, we detected a more pronounced rise of C24 
A
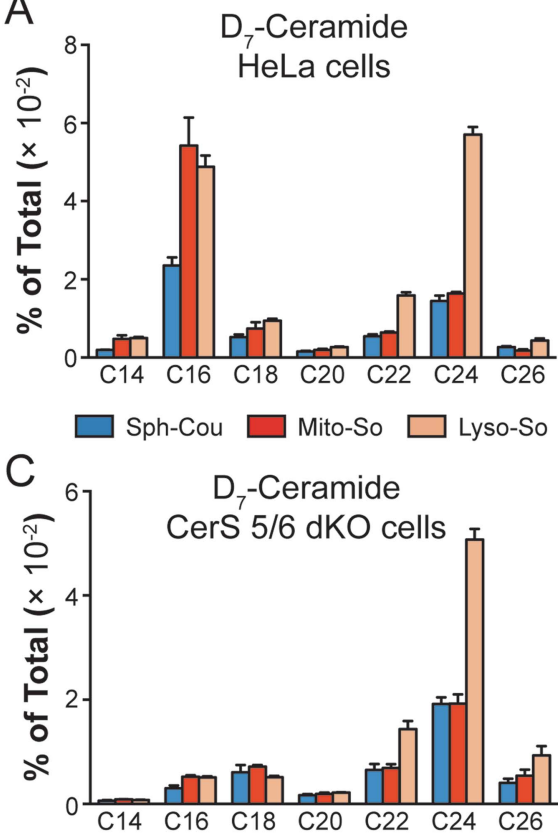
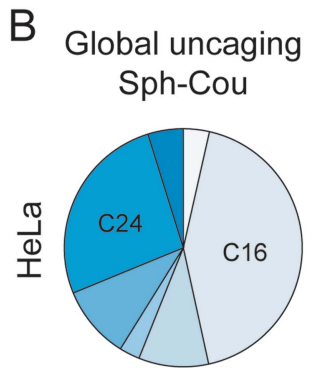

Sum: $5.47 \times 10^{-2} \%$

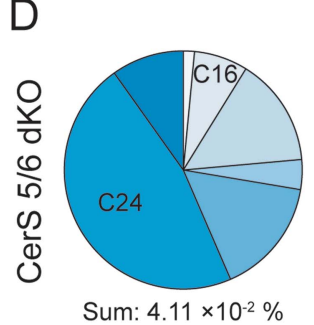

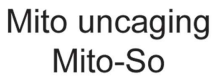

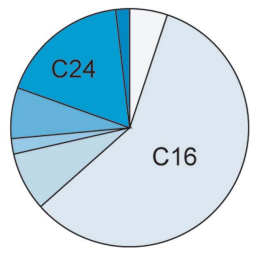

Sum: $9.29 \times 10^{-2} \%$

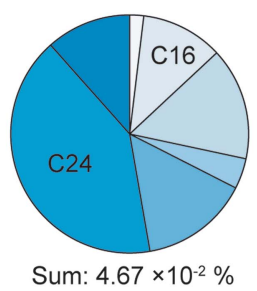

\section{Lysosome uncaging Lyso-So}

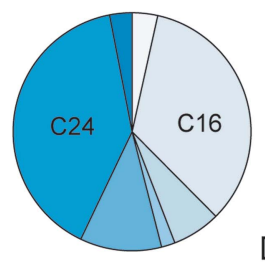

Sum: $14.3 \times 10^{-2} \%$ $D_{7}$-Cer_14 $D_{7}$-Cer_16 $D_{7}$-Cer_18 $D_{7}$-Cer_20

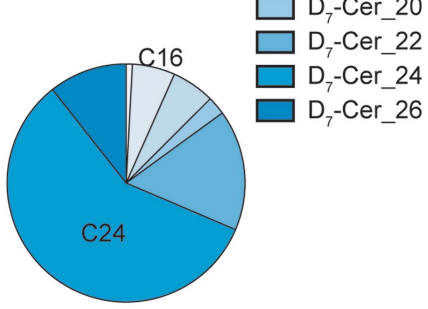

Sum: $8.74 \times 10^{-2} \%$

Fig. 4 Comparison of newly formed ceramides in wild-type (WT) and CerS 5/6 dKO cells after photoactivation. Cells were treated with caged probes $(2 \mu \mathrm{M})$, uncaged for 2 minutes, and incubated for 5 minutes prior to lipid extraction. (A and B) Analysis of $D_{7}$-ceramides in HeLa WT cells after releasing sphingosine from different sources as indicated. ( $C$ and $D$ ) Analysis of $D_{7}$-ceramides in CerS 5/6 dKO cells after releasing sphingosine from different sources as indicated. Pie charts were generated from the average values of ceramide with different acyl chains, respectively. Data were normalized by measured total lipid signals and presented as "mol\% of total". The sum indicates the summation of $D_{7}$ ceramides (\% of total) of different acyl chains. Data represent the average of three independent experiments. Error bars represent SEM.

ceramide when uncaging happened in the lysosome, and the C18 ceramide was relatively more enriched under global uncaging as well as mitochondria uncaging conditions. The combined results show that the profile of ceramide formation is greatly affected by the subcellular source of sphingosine. This is unaffected by the light-independent release of sphingosine in lysosomes because the ratio of C16/C24 ceramide does not change between 0 and 5 minutes (Fig. 3D and E). The different profiles of ceramides depending on the mitochondrial or lysosomal source were not necessarily expected because all ceramide synthases are thought to be in the endoplasmic reticulum. Thus, our data demonstrate a localization-dependent metabolic bias in ceramide synthesis, which is likely due to the transport pathways of sphingosine between the ER and other organelles.

It is well-established that lipids can serve distinct functions depending on their subcellular localization, but the experimental means to directly address this issue are limited. One major challenge lies in that small changes in lipid structures can have profound effects on crucial biological functions. ${ }^{19}$ Bifunctional lipids, ${ }^{20,21}$ with minimal chemical modification, are introduced for profiling lipid-protein interactions yet not for metabolism studies. Genetic knockout is a powerful tool, but its chronic nature compromises the results if cells find an alternative pathway, as indicated in our data for CerS $5 / 6$ dKO cells (Fig. S7E $\dagger$ ). On the other hand, our approach, which combines site-specific photoactivation, lipidomics with isotopic signatures, and genetic knockout, offers a direct way to rapidly release lipids in living cells and quantitatively analyse their metabolic conversion.

\section{Conclusions}

To summarize, we developed a lysosome-targeted photoactivation method to investigate local sphingosine metabolism. Future efforts will focus on improving the temporal control of the caged probes by finding conditions to limit the release prior to illumination. Collectively, our data showed a distinct metabolism pattern of lysosomal sphingosine, influencing the formation of S1P and specific ceramide species. These results thus unveiled a metabolic bias in ceramide synthesis dependent upon the localization of the substrate sphingosine. Combined with the mitochondrial specific release, ${ }^{9}$ this strategy offers a framework to study lipid metabolism with subcellular resolution.

\section{Conflicts of interest}

There are no conflicts to declare.

\section{Acknowledgements}

We thank the Bioimaging Center (University of Geneva) for technical assistance. T. H. was supported by the Japan Society for the Promotion of Science (JSPS) Postdoctoral Fellowships for Research Abroad. J. T. H. was supported by an EMBO/MarieCurie Long-Term Fellowship. This study was supported by the Swiss National Science Foundation (CRSII3-154405, 310030B_166686) and the NCCR Chemical Biology funded by the Swiss National Science Foundation (51NF40-160589). 


\section{Notes and references}

1 A. X. da Silveira Dos Santos, I. Riezman, M.-A. AguileraRomero, F. David, M. Piccolis, R. Loewith, O. Schaad and H. Riezman, Mol. Biol. Cell, 2014, 25, 3234-3246.

2 D. Höglinger, A. Nadler and C. Schultz, Biochim. Biophys. Acta, 2014, 1841, 1085-1096.

3 D. Subramanian, V. Laketa, R. Müller, C. Tischer, S. Zarbakhsh, R. Pepperkok and C. Schultz, Nat. Chem. Biol., 2010, 6, 324-326.

4 X. Fang, Y. Fu, M. J. C. Long, J. A. Haegele, E. J. Ge, S. Parvez and Y. Aye, J. Am. Chem. Soc., 2013, 135, 14496-14499.

5 A. Nadler, G. Reither, S. Feng, F. Stein, S. Reither, R. Müller and C. Schultz, Angew. Chem., Int. Ed. Engl., 2013, 52, 63306334.

6 D. Höglinger, P. Haberkant, A. Aguilera-Romero, H. Riezman, F. D. Porter, F. M. Platt, A. Galione and C. Schultz, eLife, 2015, 4, 300.

7 J. A. Frank, M. Moroni, R. Moshourab, M. Sumser, G. R. Lewin and D. Trauner, Nat. Commun., 2015, 6, 7118.

8 J. A. Frank, D. A. Yushchenko, D. J. Hodson, N. Lipstein, J. Nagpal, G. A. Rutter, J.-S. Rhee, A. Gottschalk, N. Brose, C. Schultz and D. Trauner, Nat. Chem. Biol., 2016, 12, 755762.

9 S. Feng, T. Harayama, S. Montessuit, F. P. David, N. Winssinger, J.-C. Martinou and H. Riezman, eLife, 2018, 7,647 .

10 C. Settembre, A. Fraldi, D. L. Medina and A. Ballabio, Nat. Rev. Mol. Cell Biol., 2013, 14, 283-296.
11 E. Lloyd-Evans, A. J. Morgan, X. He, D. A. Smith, E. ElliotSmith, D. J. Sillence, G. C. Churchill, E. H. Schuchman, A. Galione and F. M. Platt, Nat. Med., 2008, 14, 1247-1255.

12 N. Wagner, M. Stephan, D. Höglinger and A. Nadler, Angew. Chem., Int. Ed. Engl., 2018, 57, 13339-13343.

13 G. M. Strub, M. Paillard, J. Liang, L. Gomez, J. C. Allegood, N. C. Hait, M. Maceyka, M. M. Price, Q. Chen, D. C. Simpson, T. Kordula, S. Milstien, E. J. Lesnefsky and S. Spiegel, FASEB J., 2011, 25, 600-612.

14 Y. A. Hannun and L. M. Obeid, Nat. Rev. Mol. Cell Biol., 2017, 1-17.

15 M. Ikeda, A. Kihara and Y. Igarashi, Biochem. Biophys. Res. Commun., 2004, 325, 338-343.

16 M. Levy and A. H. Futerman, IUBMB Life, 2010, 347-356.

17 L. J. Siskind and M. Colombini, J. Biol. Chem., 2000, 275, 38640-38644.

18 F. A. Ran, P. D. Hsu, J. Wright, V. Agarwala, D. A. Scott and F. Zhang, Nat. Protoc., 2013, 8, 2281-2308.

19 T. Harayama and H. Riezman, Nat. Rev. Mol. Cell Biol., 2018, 19, 281-296.

20 P. Haberkant, R. Raijmakers, M. Wildwater, T. Sachsenheimer, B. Brügger, K. Maeda, M. Houweling, A.-C. Gavin, C. Schultz, G. van Meer, A. J. R. Heck and J. C. M. Holthuis, Angew. Chem., Int. Ed. Engl., 2013, 52, 4033-4038.

21 P. Haberkant, F. Stein, D. Höglinger, M. J. Gerl, B. Brügger, P. P. Van Veldhoven, J. Krijgsveld, A.-C. Gavin and C. Schultz, ACS Chem. Biol., 2016, 11, 222-230. 